\title{
Processus fondamentaux d'absorption et de relaxation de l'énergie dans les interactions laser-surface
}

\author{
G. Petite, Ph. Daguzan, S. Guizard et Ph. Martin \\ Service'de Recherche sur les Surfaces et I'Irradiation de la Matière, \\ CEA/DSM/DRECAM, CEA Saclay, 91191 Gif-sur-Yvette cedex, France
}

\begin{abstract}
Résumè : Nous discutons les processus fondamentaux d'absorption et de relaxation de l'énergie dans les interactions laser surface. Le rôle des modes collectifs dans les mètaux, et du fort couplage électron-phonon dans les isolants sont analysés en détail. Nous donnons quelques exemples de résultats récents sur l'analyse subpicoseconde du piégeage de charge dans les isolants.
\end{abstract}

\section{INTRODUCTION}

L'interaction de faisceaux laser intenses dans le domaine visible et XUV est utilisée dans un grand nombre d'applications, certaines déjà au stade industriel, et on doit pourtant admettre qu'ils reste encore un nombre appréciable de questions fondamentales auxquelles les réponses sont incertaines. Nous allons dans cet article évoquer certaines d'entre elles, et tenter de faire le point sur l'état actuel de nos connaissances sur les mécanismes d'absorption et de redistribution de l'énergie dans ce type d'interactions.

La structure électronique du matériau est évidemment un élément déterminant dans le mécanisme d'absorption, et nous verrons qu'il existe sur ce point des différences fondamentales entre les métaux d'une part et les isolants à grande bande interdite d'autre part. Les métaux sont en particulier caractérisés par l'existence de modes collectifs d'excitations dont l'importance dans les interactions laser-surface a été reconnue, mais dont les détails sont encore incertains, particulièrement dans le cas de surfaces rugueuses.

Dans le cas des isolants, le couplage électron-phonon est particulièrement fort, et nous en étudierons plusieurs conséquences aussi bien au niveau des mécanismes d'absorption que des mécanismes propres aux isolants de piégeage des excitations électroniques. Nous montrerons en particulier comment des expériences récentes utilisant des méthodes pompe-sonde en impulsions subpicoseconde ont pu apporter une grande quantité d'informations sur la cinétique, extrêmement rapide, de ces processus, qui se produisent dans des temps courts devant la durée des impulsions classiquement utilisés dans les applications au traitement ou à l'analyse laser de surfaces.

\section{INTERACTIONS LASER-METAUX : LA PHOTOEMISSION MULTIPHOTONIQUE}

Comme dans le cas des atomes, un métal soumis à un éclairement laser intense sera le siège de processus d'émission électronique même si l'énergie de photon est inférieure au travail de sortie. Les éclairements atteints avec les lasers impulsionels sont en effet largement suffisants pour que des phénomènes multiphotoniques puissent se produire. Ces émissions ont été étudiées pratiquement depuis l'apparition des lasers [1-3], mais certains aspects, pourtant classiques des études de photoémission utilisant le rayonnement synchrotron, sont restés inexplorés. Considérons la question suivante: le mécanisme dominant d'absorption d'énergie dans les interactions laser-métal repose-t-il sur des transitions directes ou indirectes? Le processus de photoémission multiphotonique est-il résonant ou non-résonant? Elles n'ont pas à notre connaissance reçu de réponse à ce jour, bien que la technique expérimentale propre à y répondre est bien connue, puisqu'il s'agit de la spectrométrie d'électrons résolue en angle.

En effet, comme le montre la figure 1, la conservation du moment cristallin implique qu'une absorption directe ne pourra se produire qu' en un nombre restreint de points du réseau réciproque (un fait 
bien connu en photo émission classique). Dans un processus multiphotonique, une résonance intermédiaire doit donc se traduire par une direction particulière d'émission. Ceci ne sera par contre pas le cas si les transitions qui dominent le processus d'émission multiphotonique sont indirectes (impliquant l'absorption ou l'émission d'un phonon). Ces dernières sont sans aucun doute moins probables qu'une transition directe résonante, mais elles sont par contre en nombre quasi-infini de sorte qu'il est impossible de dire quel est le processus qui domine en l'occurrence. L'étude de la distribution angulaire de l'émission multiphotonique devrait apporter de précieux renseignements sur ce point.

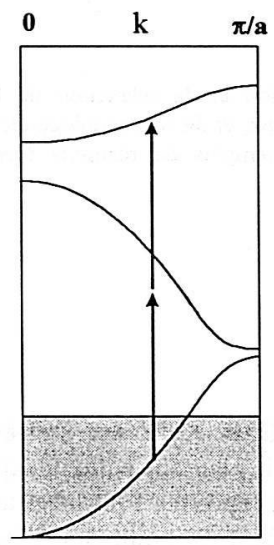

a) directe non-résonante

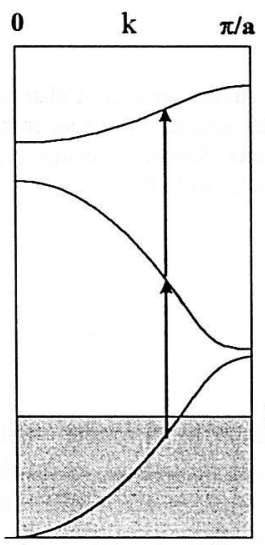

b) directe résonante

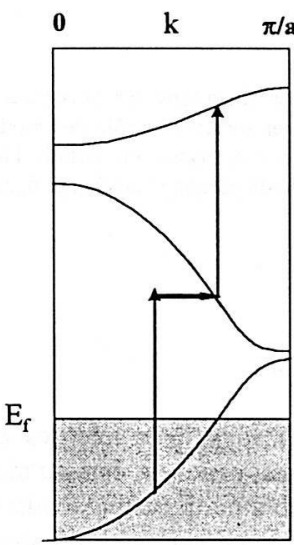

c) indirecte résonante

Figure 1 : Conservation du moment cristallin dans trois types de transitions multiphotoniques dans un métal.

Une autre caractéristique essentielle des métaux est de supporter des modes collectifs électroniques. Les oscillations plasma de volume sont l'élément dominant de l'optiques des métaux, mais l'énergie des plasmons de volume est en général trop élevée pour les lasers usuels. Mais ce n'est pas le cas pour les plasmons de surface [4], et tout indique qu'ils peuvent jouer un rôle déterminant dans l'absorption dans des expériences d'ablation par exemple, en particulier quand la surface en cause présente une certaine rugosité.

Dans le voisinage immédiat d'une interface entre un métal et le vide (ou, plus généralement un diélectrique), on peut montrer qu'il existe des oscillations localisées des électrons, qui s'éteignent rapidement que ce soit vers l'intérieur du métal ou du coté diélectrique. Ces ondes ont une nature différente suivant que la surface est plane (onde progressive, dont le vecteur d'onde est dans le plan de la surface) ou sphérique (onde stationnaire). Intéressons nous d'abord au cas plan, qui a fait l'objet des études les plus nombreuses. Pour un métal que nous caractériserons par sa fréquuence plasma $\omega_{p}$, on montre que ces ondes possèdent une relation de dispersion fixant la fréquence pour une valeur de $\mathrm{k}_{\mathrm{X}}$ donnée (l'indice $x$ signale le fait que $k$ est parallèle à la surface)

$$
k_{x}=\frac{\omega}{c} \sqrt{\frac{\varepsilon_{1}}{1+\varepsilon_{1}}}
$$

où la partie réelle de la constante diélectrique s'écrit, dans un modèle de Drude sans collisions

$$
\varepsilon_{1}=1-\omega_{p}^{2} / \omega^{2} \quad \text { d'où } \quad k_{x}=\frac{\omega}{c} \sqrt{\left(1-\omega_{p}^{2} / \omega^{2}\right) /\left(2-\omega_{p}^{2} / \omega^{2}\right)}
$$


Cette relation de dispersion comporte deux branches, l'une pour des valeurs de $\omega>\omega_{p}$, connue sous le nom de «plasmon de surface radiatif», et l'autre, pour $\omega<\omega_{\downarrow} / \sqrt{2}$, dite «plasmon de surface nonradiatif ", qui est représentée sur la figure 2 . En effet, si l'on cherche maintenant à coupler la lumière avec ces oscillations de surface, on constate que les courbes de dispersion de la lumière et du plasmon de surface non radiatif n'ont d'intersection qu'à l'origine, et il n'y a donc pas de couplage possible. Pour remédier à ce fait, on peut abaisser la courbe de dispersion de la lumière en éclairant la surface à partir d'un milieu diélectrique d'indice supérieur à celui impliqué dans l'interface. On peut par exemple déposer le métal en couche mince sur un prisme et éclairer cette couche par l'arrière (configuration de Kretschman). Il est alors possible d'exciter un plasmon de surface à l'interface métal-vide grâce à l'onde évanescente dans le métal qui à les propriétés de dispersion de la lumière dans le prisme. La résonance est alors accordée, à $\omega$ donné (par le laser) en faisant varier l'angle d'incidence (cf. figure 2).

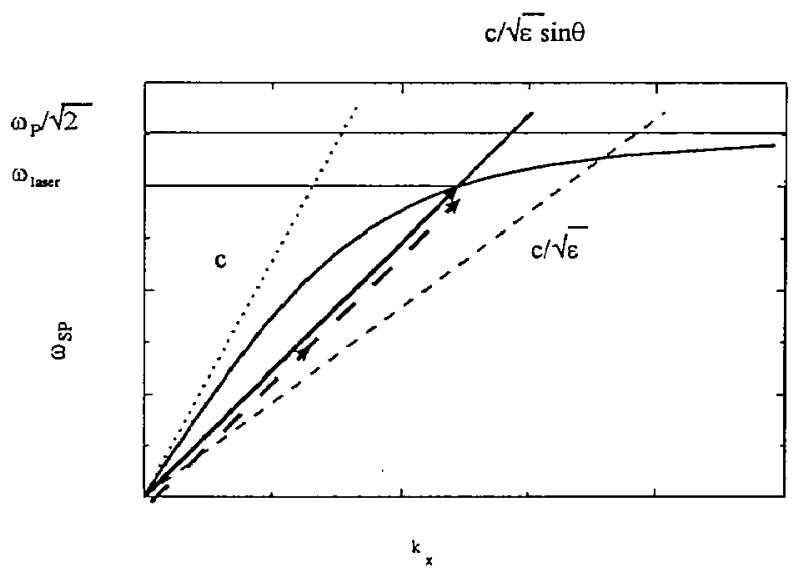

Figure 2 : relation de dispersion du plasmon de surface (courbe en trait plein) et principe du couplage par la méthode ATR. La droite en trait plein représente la courbe de dispersion de la lumière dans le prisme (constante diélectrique $\varepsilon$ ) pour une incidence $\theta$. Tous les points à gauche de la droite en tiret sur la courbe du SP peuvent donner lieu à un couplage. Noter que la lumière dans le vide ne peut se coupler puisque la droite de pente c est à gauche de la courbe de dispersion (pointillés).

Nous avons réalisé une expérience de photoémission en utilisant une couche mince d'argent déposée sur un prisme de silice en configuration de Kretschman, dont le résultat est montré sur la figure 3 . Le laser utilisé était un oscillateur au saphir dopé titane, délivrant des impulsions de $70 \mathrm{fs}$ à une cadence de 66 $\mathrm{MHz}$, et une puissance moyenne de $130 \mathrm{~mW}$. L'énergie de photon de ce laser est de $1,5 \mathrm{eV}$ (3 eV pour l'harmonique deux, que nous avons aussi utilisé) de sorte que le processus de photoémission est d'ordre trois pour la longueur d'onde fondamentale et deux pour l'harmonique. En comparant la dépendance en angle d'incidence de l'émission multiphotonique à la longueur d'onde fondamentale avec celle de la réflectivité de la couche, on constate que le couplage linéaire du champ laser au plasmon de surface est à l'origine d'une résonance importante dans le signal de photoémission (pic à $43^{\circ}$ ), ce qui avait déjà été observé [5]. On remarque aussi un pic secondaire, à l'angle de $58^{\circ}$, qui correspond précisément à l'angle de résonance pour la longueur d'onde harmonique, preuve que les plasmons de surface peuvent être impliqués dans des interactions non-linéaires. Il se pose toutefois la question de savoir s'il s'agit d'un couplage direct à deux photons entre le laser et le plasmon de surface, ou s'il s'agit d'une émission amplifiée par la faible résonance existant dans le rendement de génération d'harmonique deux à la surface de la couche quand celle ci est couplée au plasmon de surface. Des études complémentaires sont nécessaires pour éclaircir ce point. 

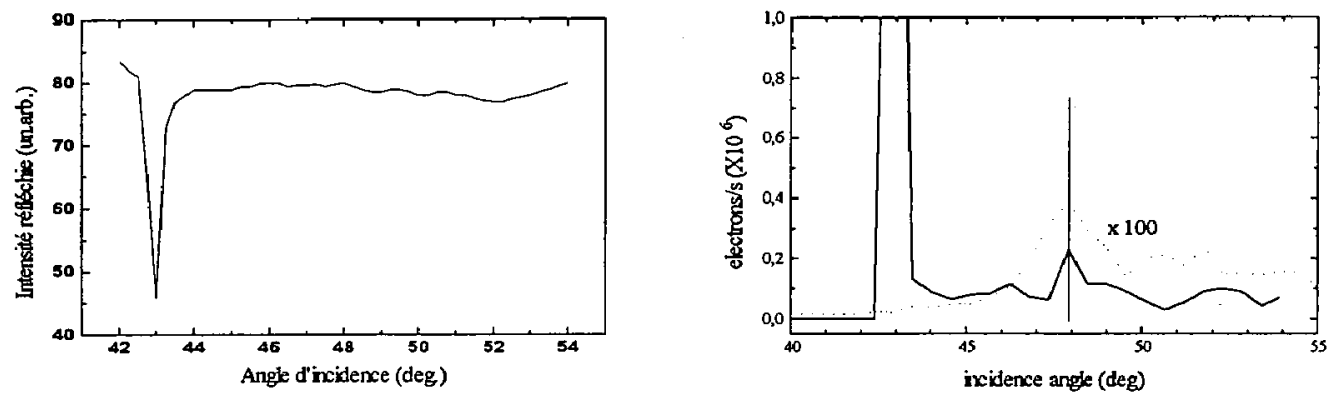

Figure 3 : gauche : absorption par plasmons de surface sur une couche mince d'argent en configuration de Kretschman $(\lambda=800 \mathrm{~nm})$. Droite : émission multiphotonique de la même couche aux longueurs d'onde de $800 \mathrm{~nm}$ (trait plein) et $400 \mathrm{~nm}$ (pointillé). Noter en particulier le pic secondaire à $800 \mathrm{~nm}$, situé à l'incidence de résonance pour $400 \mathrm{~nm}$, signe probable d'une excitation a deux photons du plasmon de surface.

Si un système comme celui de la figure 9 est un bon objet d'étude fondamentale, sa portée générale peut sembler limitée dans le cadre de l'ablation laser. Ce n'est pas le cas de la notion des plasmons de surface de systèmes sphériques (et de façon plus générale de toute surface présentant une rugosité). Dans ce cas, le couplage avec un rayonnement incident est grandement facilité par le fait qu'un plasmon de surface sphérique est, comme un plasmon de volume, une onde stationnaire ne présentant pas de dispersion, de sorte que tout rayonnement incident à la fréquence plasmon se couple sans difficulté. Dans le cas idéal d'une sphère parfaite d'un métal à électrons libres, il existe une résonance principale à $\omega_{\mathrm{p}} / \sqrt{3}$. De façon générale, on observera des résonances dont la position et la forme dépendront à la fois de la géométrie de la surface (sphère, ellipsoïde) et de la structure électronique du métal considéré (déplacements et élargissements de résonances dus au couplage plasmon de surface-transitions interbandes). De telles résonances peuvent être observées aussi bien en absorption [6] que dans des expériences d'émission électronique [7], de sorte que l'utilisation de photocathodes granulaires est actuellement à l'étude comme solution au problème de la génération d'impulsions électroniques intense par photoémission [8]. D'autres auteurs ont montré qu'une surface d'argent endommagée par un impact laser préalable émettait des ions d'énergie cinétique bien définie, égale à l'énergie du plasmon de surface [9]. Il semble bien donc que ces oscillations de surface jouent un rôle important dans la physiqque de l'ablation laser.

Sur des surfaces parfaitement définies, d'autres types d'états de surface peuvent jouer un rôle dans le processus d'émission. On a en particulier beaucoup étudié le cas des «états images » [10] qui résultent de l'interaction coulombienne attractive entre l'électron sortant et la charge image, de signe opposé, qu'il laisse derrière lui. De telles études nécessitent des conditions expérimentales extrêmement strictes, mais foumissent un bon exemple d'application au cas des solides des méthodes de spectroscopie multiphotonique classiques en physique atomique et moléculaire.

Cependant, on se rappellera que de façon générale, et bien que la profondeur de pénétration du laser puisse paraître faible au regard de l'optique, ce sont essentiellement les électrons du volume qui sont concernés par l'interaction (les effets typiques de la surface ne se manifestant que sur un petit nombre de couches atomiques). Une étude récente [11] đu comportement du courant de photoémission multiphotonique en fonction de l'angle d'incidence a par exemple montré que celui ci s'interprétait simplement à l'aide des formules de Fresnel déterminant la partie transmise du champ dans le métal. On retiendra aussi que, sauf à envisager des conditions expérimentales extrêmes (très forts éclairements, où longueur d'onde dans l'infrarouge lointain), les modèles fondés sur la théorie des perturbations interprètent de manière adéquate les observations expérimentales. Il en résulte que l'interaction entre un laser et un métal est dominée par la réponse linéaire. Des expériences particulières peuvent révéler des effets non-linéaires (photoémission multiphotonique par exemple), mais il faudra souvent prendre en compte le fait que le matériau peut évoluer sous l'effet de l'interaction au premier ordre. Le processus dominant de relaxation de l'énergie est, tout particulièrement dans le cas des électrons excités en dessous 
du seuil d'émission, le transfert d'énergie au réseau sous forme thermique. L'élévation de température qui s'en suit peut sérieusement affecter le processus d'émission électronique lui même. La théorie de FowlerDubridge, généralisée par Bechtel et al. [3] rend fort bien compte des comportements observés dans la plupart des expériences.

\section{INTERACTIONS LASER-MATERIAUX DIELECTRIQUES A GRANDE BANDE INTERDITE : PIEGEAGE DES EXCITATIONS ELECTRONIQUES}

Le cas des matériaux diélectriques à grande bande interdite est très différent de ce point de vue de celui des métaux ou des semi-conducteurs habituels. Ces matériaux sont optiquement transparents parce qu'ils ont des bandes interdites de l'ordre de 8 à $10 \mathrm{eV}$. La première excitation possible d'un électron de valence va donc nécessiter l'absorption simultanée de plusieurs photons, comme le montre la figure 4 .

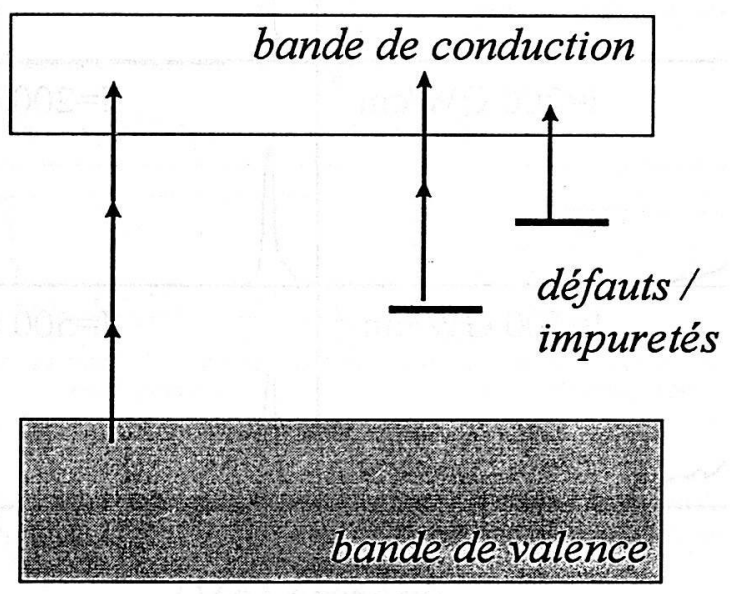

Figure 4 : différents processus d'absorptions possible dans les isolants à grande bande interdite. A gauche : intrinsèque, à droite : extrinsèque (à partir d'états de défauts)

Il faut cependant se rappeler que de tels matériaux ne sont pas parfaits, et qu'aux défauts et impuretés peuvent correspondre des états occupés dans la bande interdite, dont l'excitation peut nécessiter un nombre inférieur de photons et donc être beaucoup plus probable. Ces défauts et impuretés peuvent donc, même en concentration faible, dominer l'interaction. Les excitations intrinsèques peuvent être favorisées par l'utilisation d'éclairements élevés, et donc d'impulsions courtes (compte tenu de la fluence limite supportée par le matériau). On pourra garder en mémoire l'ordre de grandeur suivant: dans le cas de la figure 4 (transition interbande à trois photons), et pour une concentration de défauts/impuretés de l'ordre $\mathrm{du} \mathrm{ppm}$, il faudra utiliser des éclairements supérieurs à $10 \mathrm{MW} / \mathrm{cm}^{2}$ pour étudier les processus intrinsèques si le processus concernant l'impureté est d'ordre deux, et supérieurs à $10 \mathrm{GW} / \mathrm{cm}^{2}$, si il est d'odre un [12].

Comme dans le cas des métaux, l'absorption de photons par les électrons de conduction est possible dans les isolants excités par laser. Cette absorption peut être soit directe soit, et c'est le plus souvent le cas, indirecte. En effet ces transitions, dans lesquelles un photon est absorbé dans une collision électron phonon, sont d'autant plus probables que le taux de collision est fort, ce qui est le cas dans les matériaux de ce type (de l'ordre de $10^{15} \mathrm{~s}^{-1}$ ). Une expérience récente a mis en évidence ce type d'absorption par l'étude des spectres de photoémission d'échantillons de quartz- $\alpha$ sous irradiation laser interise [13]. La figure 5 montre deux séries de tels spectres obtenus pour des éclairements laser de 50 à $500 \mathrm{GW} / \mathrm{cm}^{2}$ à deux longueurs d'ondes $(620$ et $310 \mathrm{~nm}$ ). On constate que pour $620 \mathrm{~nm}$, les spectres aux grands 
éclairements comportent des électrons d'énergie allant jusqu'à $8 \mathrm{eV}$, soit près de quatre fois l'énergie du photon $(2 \mathrm{eV})$. Cet effet disparaît dans ce cas (impulsions subpicoseconde) à $310 \mathrm{~nm}$. Le caractère séquentiel de la série d'absorptions est bien mis en évidence en utilisant au lieu d'impulsions de $70 \mathrm{fs}$, comme dans le cas de la figure 5 , des impulsions de $30 \mathrm{ps}$, à la longueur d'onde de $355 \mathrm{~nm}$. L'absorption d'un photon supplémentaire est alors clairement visible dès un éclairement de $20 \mathrm{GW} / \mathrm{cm}^{2}$ [14], auquel aucun effet n'est mesuré même dans le visible avec une impulsion subpicoseconde, ce qui révèle bien un effet important de la durée de l'impulsion.

(a) $620 \mathrm{~nm}$

(b) $310 \mathrm{~nm}$

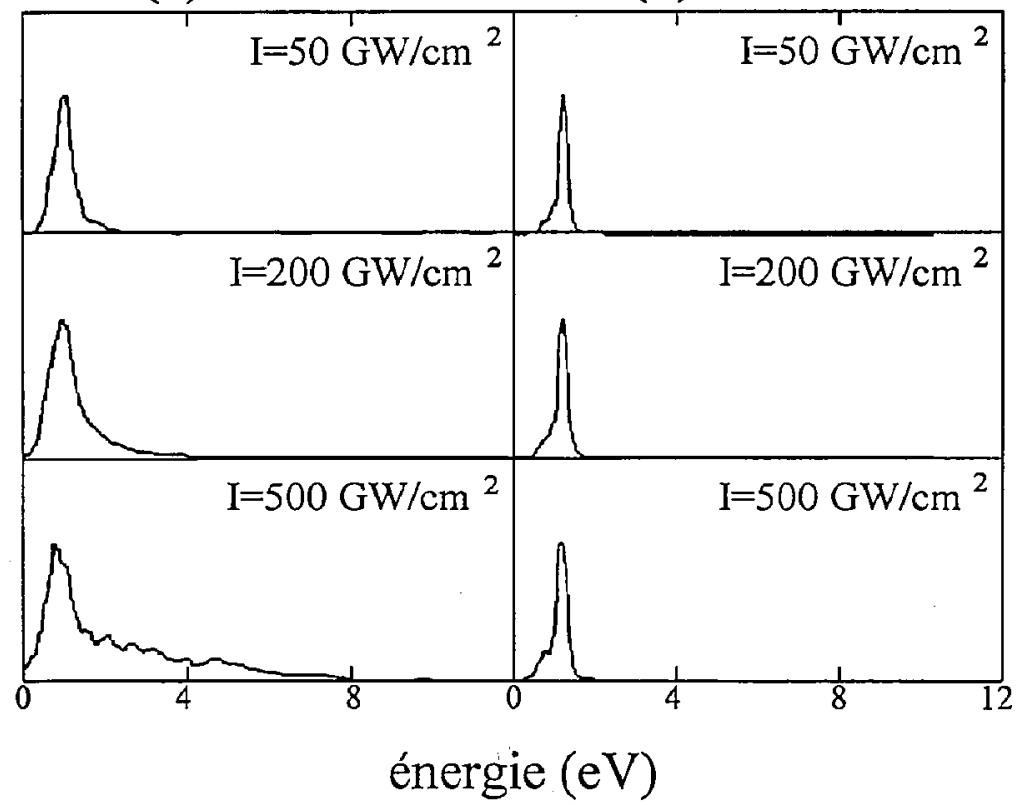

figure 5 :-Evolution des spectres de photoélectrons dans le quartz- $\alpha$ en fonction de l'éclairement laser, pour deux longueurs d'onde différentes ((a) $620 \mathrm{~nm}$ et (b) $310 \mathrm{~nm}$ ). Durée de l'impulsion : $70 \mathrm{fs}$

Une autre caractéristique essentielle des isolants est leur capacité à piéger les charges. Contrairement à ce qui se passe dans un métal, où toute l'énergie absorbée est finalement dépensée sous forme de chaleur (en dessous du seuil d'ablation, il s'entend), une partie de cette énergie va se retrouver stockée dans le réseau sous forme "d'énergie de relaxation» (une déformation du réseau cristallin associée à la charge piégée). Compte tenu de l'importance de ces phénomènes vis à vis de la création de défauts d'irraḑiation, ils ont été largement étudiés, et en particulier leurs aspects cinétiques.

Il est possible, en utilisant une méthode interférentielle fonctionnant dans le domaine des fréquences [15] d'étudier les modifications induites par une excitation électronique de l'indice de réfraction d'un matériau, et ce avec une résolution temporelle de l'ordre de $100 \mathrm{fs}$. Le résultat d'une telle expérience [16] sur trois oxydes isolants $\left(\mathrm{SiO}_{2}, \mathrm{Al}_{2} \mathrm{O}_{3}\right.$ et $\left.\mathrm{MgO}\right)$ est montré sur la figure 6 . Le pic positif aux petits délais est du à l'effet Kerr (modification par la pompe intense de l'indice à la fréquence sonde) et subsiste tant que les impulsions pompe et sonde sont présentes ensemble dans le matériau. L'abaissement de l'indice que l'on détecte après dans tous les cas (déphasage négatif) est du à la contribution des électrons de conduction (que l'on peut décrire par un modèle de Drude) et le déphasage positif que l'on voit aux temps longs dans le cas de $\mathrm{SiO}_{2}$, est produit par les électrons piégés dans des niveaux profonds dans la bande de conduction (à peu prés au milieu de la bande 
interdite). Il est intéressant de constater que des matériaux apparemment similaires ont du point de vue du piégeage des électrons de conduction des comportement très différents: piégeage extrêmement rapide dans $\mathrm{SiO}_{2}$ (temps moyen $150 \mathrm{fs}$ ), et plus lent de presque trois ordres de grandeur dans $\mathrm{Al}_{2} \mathrm{O}_{3}$ et $\mathrm{MgO}$ ! On a pu montrer dans $\mathrm{SiO}_{2}$ que le piégeage des électrons était du à la formation d'un défaut transitoire (l'exciton auto-piégé), constitué par un électron piégé sur un atome de silicium et un trou piégé sur un oxygène voisin.
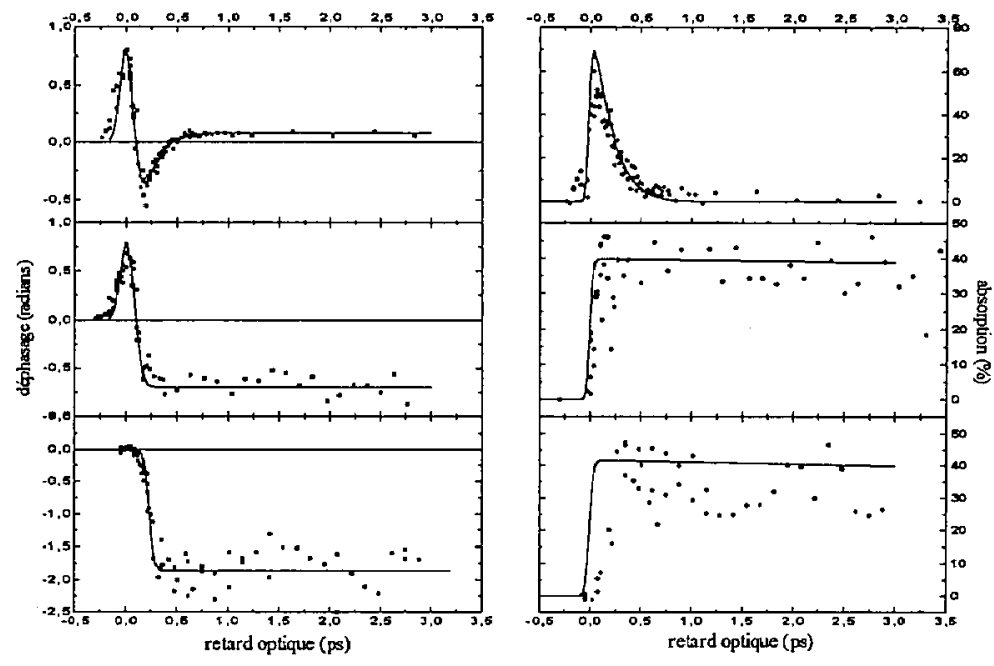

figure 6 : déphasage (gauche) et absorption (droite) subie par une impulsion sonde en fonction du délai pompe/sonde pour (de haut en bas ) $\mathrm{SiO}_{2}, \mathrm{Al}_{2} \mathrm{O}_{3}$ et $\mathrm{MgO}$. La référence est donnée par un échantillon non-irradié : un déphasage négatif (positif) correspond donc à un indice inférieur (supérieur) à $\mathrm{n}_{0}$.

On notera de plus qu'à la présence d'électrons dans la bande de conduction est associée une absorption importante, dont l'origine physique est la même que celle des électrons rapides mesurés sur la figure 5. On trouve même un excellent accord quantitatif entre la valeur mesurée de l'absorption et les sections efficaces de collision electron-photon-phonon qui permettent de simuler les spectres de la figure 5 .

Le temps d'apparition de ce défaut à pu être mesuré dans une expérience d'absorption résolue en temps [17], au pic de sa bande d'absorption à $5.2 \mathrm{eV}$. L'évolution de cette absorption est montrée sur la figure 7, et on constate que le temps d'apparition du défaut est identique au temps de piégeage des électrons de conduction, une preuve de la corrélation entre ces deux phénomènes.

L'exciton auto-piégé, dont la cinétique de formation est étudiée ici, est à l'origine de la formation de centres colorés dans les matériaux optiques, et ces phénomènes associés au piégeage d'excitations électroniques sont d'une extrême importance pour la compréhension de l'interaction entre un laser intense et des matériaux optiques. De façon plus générale, ce problème est commun à toutes les irradiations opérant par dépôt d'énergie électronique, et en particulier les rayons X. L'utilisation de lasers comme source d'excitation est pour le moment la seule méthode pour aborder les études de cinétique de piégeage avec la résolution temporelle nécessaire qui, nous venons de le voir, est extrêmement courte (de l'ordre de $10^{-13} \mathrm{~s}$ au moins). Les deux méthodes exposées ci-dessus montrent bien la puissance des méthodes optiques pour l'étude de ces phénomènes. 


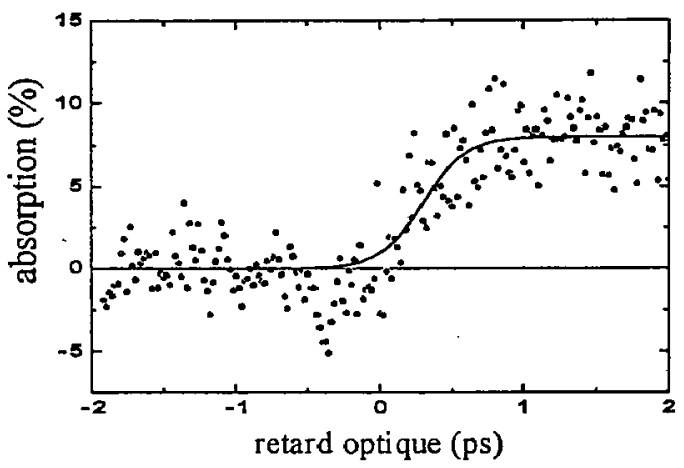

figure 7 : absorption transitoire à $5,2 \mathrm{eV}$ d'un échantillon de quartz- $\alpha$ suite à une irradiation laser intense. Le défaut responsable de cette absorption est l'exciton auto-piégé, dont le temps de formation mesuré ici est identique au temps de piégeage de électrons de conduction mesuré fig. 6

De ce point de vue, la possibilité de disposer d'impulsions XUV intenses et sub-picosecondes, parfaitement synchronisées avec le laser servant à les produire (génération d'harmoniques d'ordre élevé) offre l'opportunité d'étendre ce type d'études des cinétiques de formation de défauts à cette gamme de longueur d'onde d'excitation.

[1] Gy. Farkas, Zs Naray and P. Varga, Phys. Lett. 24 A, 134 (1967); Gy. Farkas and Z. Gy. Horvath, Opt. Comm. 12, 392 (1974)

[2] E.M. Logothetis and P.L. Hartmann, Phys. Rev. 187, 460 (1969)

[3] J.H. Bechtel, W.L. Smith and N. Bloembergen, Opt. Comm. 13, 56 (1975), Phys. Rev. B 15, 4557 (1977)

[4] H. Raether, « Surface Plasmons ", Springer-Verlag (1986)

[5] T. Tsang, T. Srinivasan-Rao and J. Fisher, Phys. Rev. B, 43, 8870 (1991)

[6] H. Monard, Thèse, Université Paris VI (1996),

[7] P. Montchicourt, Communication Privée, publication en cours.

[8] F. Sabary et H. Bergeret, Nucl. Inst. Meth. Phys. Research, A340, 199 (1994)

[9] M. Shea et R. Compton, in "Laser Ablation ", Lect. Notes in Phys., 389, (Springer) p. 234 (1991)

[10] W. Steinman, Appl. Phys. A, 49, 365 (1989)

[11] A. Damascelli, G. Gabetta, A. Lumachi, L. Fini, F. Parmigiani, Phys. Rev. B, 54, 6031 (1996)

[12] S. Guizard, P. Martin and G. Petite, J. Phys. Condens. Matter 5, 7033 (1993)

[13] P. Daguzan, S. Guizard, K. Krastev, P. Martin, G. Petite, A. Dos-Santos et A. Antonetti, Phys. Rev. Lett., 73, 2352 (1994)

[14] P. Daguzan, S. Guizard, P. Martin, G. Petite, A. Dos-Santos et A. Antonetti, J.Opt. Soc.Am. B, 13, 138 (1996)

[15] P. Audebert, Ph. Daguzan, A. Dos Santos, J. C. Gauthier, J.P. Geindre, S. Guizard, G. Hamoniaux, K. Krastev, P. Martin, G. Petite, and A. Antonetti, Phys. Rev. Lett. 73, 1990 (1994).

[16] S. Guizard, P. Martin, Ph. Daguzan, G. Petite, P. Audebert, J. P. Geindre, A. Dos Santos, and A. Antonetti, Europhys. Lett. 29, 1995 (1995).

[17] S.Guizard, P. Martin, G. Petite, P. D'Oliveira and P. Meynadier ; J. Phys. C : Condens. Matter 8, 1281 (1996) 